Article

\title{
Reducing Pup Litter Size Alters Early Postnatal Calcium Homeostasis and Programs Adverse Adult Cardiovascular and Bone Health in Male Rats
}

\author{
Jessica F. Briffa ${ }^{1}$, Rachael O’Dowd ${ }^{1}$, Tania Romano ${ }^{1,2}{ }^{,}$Beverly S. Muhlhausler ${ }^{3}$, \\ Karen M. Moritz ${ }^{4}$ and Mary E. Wlodek ${ }^{1, *}$ \\ 1 Department of Physiology, The University of Melbourne, Parkville 3010, Australia; \\ jessica.griffith@unimelb.edu.au (J.F.B.); odowd.rachael.a@edumail.vic.gov.au (R.O.); \\ t.romano@latrobe.edu.au (T.R.) \\ 2 Department of Physiology, Anatomy and Microbiology, LaTrobe University, Bundoora 3083, Australia \\ 3 Department of Food and Wine Science, School of Agriculture, Food and Wine, FOODplus Research Centre, \\ The University of Adelaide, Adelaide 5064, Australia; Beverly.Muhlhausler@adelaide.edu.au \\ 4 Child Health Research Centre and School of Biomedical Sciences, The University of Queensland, \\ St. Lucia 4101, Australia; k.moritz1@uq.edu.au \\ * Correspondence: m.wlodek@unimelb.edu.au; Tel.: +61-3-8344-8801
}

Received: 3 December 2018; Accepted: 3 January 2019; Published: 8 January 2019

\begin{abstract}
The in utero and early postnatal environments play essential roles in offspring growth and development. Standardizing or reducing pup litter size can independently compromise long-term health likely due to altered milk quality, thus limiting translational potential. This study investigated the effect reducing litter size has on milk quality and offspring outcomes. On gestation day 18 , dams underwent sham or bilateral uterine vessel ligation surgery to generate dams with normal (Control) and altered (Restricted) milk quality/composition. At birth, pups were cross-fostered onto separate dams with either an unadjusted or reduced litter size. Plasma parathyroid hormone-related protein was increased in Reduced litter pups, whereas ionic calcium and total body calcium were decreased. These data suggest Reduced litter pups have dysregulated calcium homeostasis in early postnatal life, which may impair bone mineralization decreasing adult bone bending strength. Dams suckling Reduced litter pups had increased milk long-chain monounsaturated fatty acid and omega-3 docosahexaenoic acid. Reduced litter pups suckled by Normal milk quality/composition dams had increased milk omega- 6 linoleic and arachidonic acids. Reduced litter male adult offspring had elevated blood pressure. This study highlights care must be taken when interpreting data from research that alters litter size as it may mask subtle cardiometabolic health effects.
\end{abstract}

Keywords: reduced litter size; postnatal calcium homeostasis; adult bone health; milk composition

\section{Introduction}

It is well known that the in utero and early postnatal environments play crucial roles in offspring growth, development and long-term health. David Barker first demonstrated a causal link between size at birth and later-life cardiovascular disease [1,2], which has been since expanded to include several adverse pregnancy perturbations, including fetal growth restriction $[3,4]$, maternal undernutrition $[5,6]$, maternal alcohol consumption $[7,8]$ and maternal stress $[9,10]$. Postnatal growth rate and development is directly proportional to the quality and quantity of milk produced and is particularly influenced by fatty acid composition [11]. Specifically, high intakes of omega-3 fatty acids in early postnatal life is associated with reduced fat deposition and improved cardiometabolic health $[12,13]$. 
More recent experimental studies have demonstrated that altered maternal nutrition during the lactation period can program adult offspring cardiometabolic disease. Specifically, pups suckled by dams fed a cafeteria diet during lactation exhibit a "thin-outside-fat-inside phenotype" (lean with increased abdominal fat) and impaired metabolic health in adulthood [14]. Whereas, male offspring cross-fostered onto a dam fed an isocaloric low-protein diet (6\% protein) throughout pregnancy and lactation have increased blood pressure and renal dysfunction $[15,16]$. As milk is the sole source of nutrition during early postnatal life, these studies strongly support a role for altered milk quality and/or quantity as a mechanism through which maternal nutritional status during lactation influences offspring disease.

In the developmental programming field, many researchers standardize or reduce litter size at birth to normalize milk intake across pups and cohorts [17], with the degree of litter size reduction dependent on the experimental model and research question [18-25]. A previous study identified that pup body weight and development during lactation is dependent on litter size at birth [26], which the authors hypothesize is likely due to mutual maternal and offspring adjustment to a genetically determined litter size [26]. This suggests that studies that cull pups to standardize litter size have the potential to disturb this biological process and that the original litter size may continue to have some influence on offspring development. In line with this, we previously demonstrated that reducing the litter size of healthy Wistar Kyoto (WKY) rat dams at birth (from 10-14 pups per litter to 5 pups per litter) decreases offspring body weight during early life and increases adult male blood pressure likely due to mesenteric artery stiffness and compromises bone health [27-31]. Whereas, severely reducing litter size to 3 pups (inducing early postnatal overnutrition) increases offspring body weight by weaning, alters cardiac structure and function and programs poor metabolic health [32,33]. Thus, there is a need to better understand the impact of reducing litter sizes to differing extents on the subsequent outcomes of the pups.

The mechanism behind this disease programming due to reducing litter size is likely due to its effect on milk composition. Specifically, we have recently demonstrated that both the dam and pup can modulate the maternal milk composition [34]. Thus, dams suckling growth restricted pups have improved milk fatty acid composition (characterized by increased LC-polyunsaturated fatty acid (PUFA) and LC-monounsaturated fatty acid (MUFA)), which is likely to be a compensatory mechanism aimed at supporting pup growth and organ development [34]. This finding suggests that the decreased pup milk intake, rather than poor milk quality, results in the aforementioned long-term disease [27-29]. Therefore, it is possible that reducing litter size at birth to 5 pups (similarly to the reduction in litter size we observe following uteroplacental insufficiency surgery) can itself induce changes in the early postnatal environment that has consequences for adult health outcomes and may thus mask or exacerbate any offspring outcomes due to adverse pregnancy and/or lactational events.

Therefore, the aim of this study was to investigate the effect of reducing litter size at birth on pup postnatal growth and development, mammary development and maternal milk composition. We additionally characterized if there were sex-specific differences in long-term cardiometabolic and bone health outcomes due to reducing litter size at birth and whether these outcomes were exacerbated if the pup was suckled by a dam with altered milk quality/composition.

\section{Materials and Methods}

\subsection{Animals}

All experiments were approved by The University of Melbourne's animal experimentation ethics sub-committee (AEC: 02081) following the National Health and Medical Research Councils (NHMRC) Australian code for the care and use of animals for scientific purposes. Female WKY rats ( 9 to 13 weeks of age) were obtained from the Animal Resources Centre (Canning Vale, WA, Australia) and provided with a 12-h light/dark cycle at $19-22^{\circ} \mathrm{C}$ with ad libitum access to food and water. To generate rats with 'normal' and 'altered' milk quality/composition, rats were mated and surgery 
performed on day 18 of gestation (term $=22$ days) as described previously [35]. Briefly, F0 pregnant rats were randomly allocated to a sham (Normal milk quality/composition; Control; $n=7-8$ per group) or uteroplacental insufficiency (Altered milk quality/composition; Restricted; $n=8$ per group) group, that experience premature lactogenesis [30], and were anaesthetized with $4 \%$ isoflurane and $650 \mathrm{~mL} / \mathrm{min}$ oxygen flow (reduced to $3.2 \%$ isoflurane and $250 \mathrm{~mL} / \mathrm{min}$ oxygen flow when suturing to aid in the animals recovery) to reduce the duration of anesthetic exposure and aid in recovery [35]. Rats were then allowed to deliver naturally (Figure 1a). Pups from the sham (Control) operated dams were cross-fostered 1 day after birth (PN1) randomly onto separate dams (Control or Restricted) where the litter size was unaltered from the surrogate (Standard) or reduced to 5 pups (Reduced) giving rise to four experimental groups (Figure 1b); Standard litter size suckled by Normal milk quality/composition dams (mean litter size 10.38; range 9-12 pups), Reduced litter size suckled by Normal milk quality /composition (mean litter size 5.00; range 5 pups), Standard litter size suckled by Altered milk quality/composition dams (mean litter size 11.50; range 7-15 pups) and Reduced litter size suckled by Altered milk quality/composition (mean litter size 6.00; range 3-9 pups) with $n=15-18$ dams per group [17,31,36-38]. Birth weight (3.5 $\pm 0.4 \mathrm{~g}$ vs. $4.0 \pm 0.04 \mathrm{~g}$ for Restricted and Control dams, respectively) and litter size ( $8.5 \pm 0.4$ pups vs. $11.1 \pm 0.4$ pups for Restricted and Control dams, respectively) of pups born to ligation surgery (Restricted; Altered milk quality/composition) dams was reduced compared to sham-operated (Control; Normal milk quality/composition) dams.

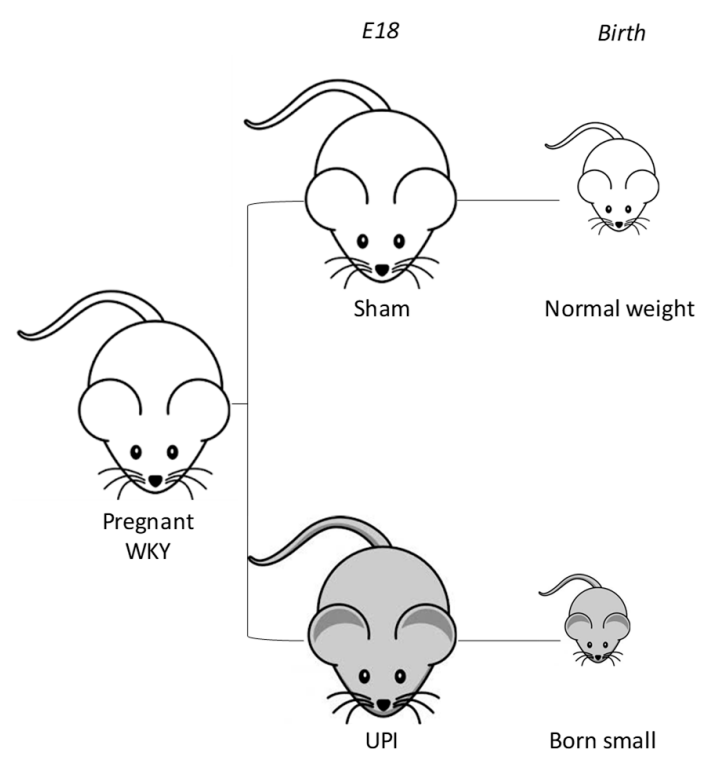

(a)

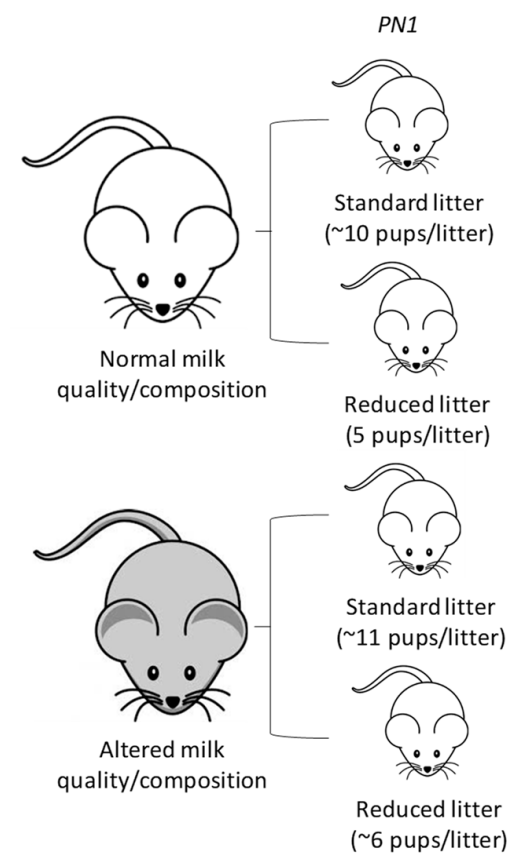

(b)

Figure 1. Study design. (a) Pregnant Wistar Kyoto (WKY) rats underwent Sham (white) or uteroplacental insufficiency (UPI; grey) surgery on day 18 of gestation (E18) and allowed to deliver naturally at birth. (b) On postnatal day 1 (PN1) pups from the Sham surgery dams were cross-fostered onto separate Sham (with Normal milk quality/composition) or UPI (with Altered milk quality/composition) dams with either an intact or reduced litter size.

\subsection{Study 1: Postnatal Study}

On the morning of PN6, one cohort of pups were removed from their dam and weighed, killed via decapitation, and blood was collected and pooled within litters. Aprotinin (Sigma-Aldrich; Castle Hill, NSW, Australia) was added to the tubes used for plasma PTHrP analysis [39]. One pup per litter was frozen whole and stored at $-20^{\circ} \mathrm{C}$ for whole body calcium analysis. Pup stomach contents were 
collected for fatty acid analysis. Dams were anaesthetized (Ketamine $(50 \mathrm{mg} / \mathrm{kg}$; Parnell Laboratories, Alexandria, NSW, Australia) and Ilium Xylazil-20 (10 mg/kg; Troy Laboratories, Glendenning, NSW, Australia)) 4-6 h after removal of the pup to allow milk to accumulate and milk was collected following gentle massage of the left mammary gland and teats without the need for hormonal stimulation [40], then euthanized by cardiac puncture with blood collected for subsequent analysis. The mammary glands were dissected, weighed and the right mammary gland immediately snap frozen in liquid nitrogen or fixed in 10\% neutral buffered formalin (Perrigo; Balcatta, WA, Australia) for histological analysis.

\subsubsection{Mammary ‘Real-Time' PCR and Histology}

'Real-time' PCR was used to quantitate milk protein gene expression in mammary tissue on PN6 as described previously with $n=3-7$ dams per group [30]. Briefly, RNA was extracted from mammary tissue using the Polytron PT 3100 (Biolab; Clayton, VIC, Australia) homogenizer and a commercially available kit (RNeasy Lipid Tissue Mini Kit from Qiagen; Chadstone, VIC, Australia). RNA was then DNase treated using the Ambion DNA free kit (Life Technologies; Mulgrave, VIC, Australia). First strand cDNA was generated from $1 \mu \mathrm{g}$ RNA using the Superscript II Single Stranded cDNA kit (Life Technologies). qPCR was performed then conducted against the milk protein genes Pthrp, b-casein and a-lactalbumin with Ribosomal $18 \mathrm{~S}$ as the reference gene [34]. The reaction was activated by heating the mixture to $95^{\circ} \mathrm{C}$ for $10 \mathrm{~min}$, then 'Real-time' PCR reactions were ran for 40 cycles of $95^{\circ} \mathrm{C}$ for $15 \mathrm{~s}$ and $60^{\circ} \mathrm{C}$ for $60 \mathrm{~s}$. For relative quantification of gene expression, a multiplex comparative threshold cycle method was employed [35]. $18 \mathrm{~S}$ values were not different between treatments.

Fixed mammary tissue was processed into paraffin blocks, sectioned at $5 \mu \mathrm{m}$ and stained with hematoxylin and eosin ( $n=4-5$ per group). Five sections per sample were analyzed for alveolar area and number using ImagePro Software (Medai Cybernetics; Warrendale, PA, United States of America) [40].

\subsubsection{PTHrP, Corticosterone, Calcium and Electrolyte Measurements}

Plasma, milk and mammary tissue concentrations of PTHrP were quantified by a N-terminal radioimmunoassay with a minimum detection limit of $2 \mathrm{pmol} / \mathrm{L}$, and intra- and inter-assay coefficients of variation of $4.8 \%$ and $13.6 \%$ respectively with $n=7-9$ per group [41]. Plasma corticosterone was measured by enzyme immunoassay validated for direct measurements in diluted plasma following the manufacturer's protocol (Cayman Chemical; Ann Arbor, MI, United States of America) with a minimum detection limit of $30 \mathrm{pg} / \mathrm{mL}$, and intra- and inter-assay coefficients of variation of $7.4 \%$ and $7.0 \%$ respectively ( $n=4-8$ per group). Total calcium concentrations were determined using colorimetric spectrometry using the Synchron CX-5 Clinical System (Beckman Coulter; Lane Cove, NSW, Australia) and ionic calcium (active or free calcium; regulated by PTHrP), sodium and potassium concentrations were determined using ion selective electrodes correcting for $\mathrm{pH}$ (Ciba-Corning model 644; Cambridge, MA, United States of America) from milk as well as from pup and maternal plasma $(n=5-8$ per group) $[39,42]$. Total calcium concentration in the pup body was determined after ashing using the CX-5 Analyzer ( $n=7-9$ per group) [30,40]. Total protein and lactose concentrations were analyzed as described previously with $n=4-6$ per group [30].

\subsubsection{Fatty Acid Analysis}

Total fatty acid composition of the milk was determined by the direct trans-esterification method of Lepage and Roy [43] as previously described [34] with $n=6-7$ dams per group. Briefly, 50 to $100 \mu \mathrm{L}$ of milk was placed into a screw-capped Teflon-lined tube containing C23:0 as an internal standard. After the 1-h trans-esterification procedure and recovery of the fatty acid methyl esters (FAMEs) in the benzene phase, the FAMEs were analyzed by capillary gas liquid chromatography. FAMEs were then separated and measured on a Shimadzu (17A) gas chromatograph with flame ionization detection. A $50 \mathrm{~mm} \times 0.25 \mathrm{~mm}$ BPX-70 fused silica capillary column (SGE Scientific; Ringwood, VIC, Australia) 
with a film thickness of $0.25 \mu \mathrm{m}$ was used in conjunction with a Shimadzu on-column auto-injector. Ultrahigh purity hydrogen was used as a carrier gas at a flow rate of $2 \mathrm{~mL} / \mathrm{min}$. A temperature gradient program was used with an initial temperature of $170^{\circ} \mathrm{C}$, increasing at $3{ }^{\circ} \mathrm{C} / \mathrm{min}$ to $218^{\circ} \mathrm{C}$. Identification of the FAMEs was made by comparison with the retention times of chromatography reference standard mixtures (Nu-Chek Prep; Elysian, MN, United States of America) [44].

\subsection{Study 2: Lactation and Adult Study}

Another cohort of pups stayed with their cross-fostered dam until weaning (PN35) and were weighed from birth (PN1) to weaning (PN3, PN6, PN10, PN14, PN17, PN21, PN24, PN28 and PN35) to determine pup growth and milk intake. Pups were weighed during a 1-h maternal separation period followed by a 3-h re-feeding period. Milk intake was calculated as a percentage of pup body weight gain after feeding compared to pre-feeding [30] and the area under the milk intake curve was calculated between PN3 and PN17 as an index of milk consumption. After weaning, male and female pups were housed separately. At 6 months, offspring ( 1 male and 1 female per litter) underwent an intra-arterial glucose tolerance test (IAGTT) and tail-cuff blood pressure measurements as previously described [36-38]. Rats were then weighed and anaesthetized with an intraperitoneal injection (50 mg/ $\mathrm{kg}$ Ketamine and $10 \mathrm{mg} / \mathrm{kg}$ Ilium Xylazil-20) and the right hind limbs were collected; all females underwent post-mortem when they were in estrous.

\subsubsection{Bone Analyses}

The right hind limb was dissected to separate the femur bone from soft tissue and femur length measured using digital calipers ( $n=9-19$ per group per sex). Individual femurs then underwent peripheral quantitative computed tomography (pQCT) to measure bone volumetric content, density and stress strain index using methods previously described [31,45-47]. Briefly, two slices of $1 \mathrm{~mm}$ thickness (voxel size $0.1000 \mathrm{~mm}^{3}$, peel mode 20, contour mode 1) were taken at distances of $15 \%$ and $50 \%$ from the reference line to quantify both trabecular and cortical bone tissue, respectively. A tissue density of $280 \mathrm{mg} / \mathrm{cm}^{3}$ or less was identified as trabecular bone, whereas a density of $710 \mathrm{mg} / \mathrm{cm}^{3}$ was representative of cortical bone. Automatic density thresholding $\left(400 \mathrm{mg} / \mathrm{cm}^{3}\right)$ was used to eliminate the effects of any soft tissue which may have remained on the femur after dissection. Bone mineral content, density, and stress strain index (index of bone bending strength) were then measured.

\subsubsection{Plasma Analyses}

Plasma were analyzed for glucose using enzymatic fluorometric analysis and insulin using a rat insulin radioimmunoassay kit (Merck Millipore; Bayswater, VIC, Australia) as previously described $(n=6-11$ per group per sex) [38,48]. Fasting plasma glucose and insulin was taken as the average of two-time points (10 and $5 \mathrm{~min}$ before injection). First-phase insulin secretion was calculated as the incremental area under the insulin curve between 0 and $5 \mathrm{~min}$ after the intra-arterial injection of glucose. Homeostasis model assessment for insulin resistance (HOMA-IR) was then determined [38].

\subsection{Statistical Analysis}

Data were analyzed using a two-way ANOVA to determine differences between Pup (Standard or Reduced litter size) and Dam (Normal or Altered milk quality/composition) groups. If an interaction was present in the two-way ANOVA, a Tukey's post-hoc test was performed to identify any effect of reducing litter size within each maternal group and any effect altering maternal milk quality/composition has on pup outcomes based on litter size. Where appropriate data analysis was performed on each sex. ANOVA statistical analysis was performed using SPSS Statistics 22 (IBM; St Leondards, NSW, Australia). All data are presented as mean \pm SEM and a $p<0.05$ was assessed as being statistically significant. 


\section{Results}

\subsection{Growth to Weaning}

As mentioned previously, dams that underwent uteroplacental insufficiency surgery gave birth to pups that were growth restricted. Not surprisingly, however, birth weight in the four experimental groups was not different (as only offspring from Sham-operated dams were utilized in the study) and litter size was decreased in the groups that had their litter size Reduced at birth on PN1 and PN6, with no differences in cannibalism across groups by PN6 (Table 1). From PN6 male (all ages) and female (except for PN14, PN21 and PN28) body weight was decreased ( $-6 \%$ and $-7 \%$ for males and females, respectively) if they were suckled by a dam with Altered milk quality/composition. Body weight at weaning was not, however, different between groups (PN35; Table 1).

Table 1. Cross-fostered litter size at postnatal day (PN) 6 and body weight from birth (PN1) to weaning (PN35) ( $n=12-18$ litter averages per group).

\begin{tabular}{|c|c|c|c|c|c|c|c|c|}
\hline \multirow{2}{*}{\multicolumn{2}{|c|}{ Pup-on-Dam }} & \multirow{2}{*}{$\begin{array}{l}\text { Standard- } \\
\text { on-Normal }\end{array}$} & \multirow{2}{*}{$\begin{array}{l}\text { Standard- } \\
\text { on-Altered }\end{array}$} & \multirow{2}{*}{$\begin{array}{l}\text { Reduced- } \\
\text { on-Normal }\end{array}$} & \multirow{2}{*}{$\begin{array}{l}\text { Reduced- } \\
\text { on-Altered }\end{array}$} & \multicolumn{3}{|c|}{ Two-Way ANOVA } \\
\hline & & & & & & Pup & Dam & Interaction \\
\hline \multicolumn{9}{|c|}{ Litter Size } \\
\hline \multicolumn{2}{|c|}{ PN1 } & $10.4 \pm 0.4$ & $11.5 \pm 0.9$ & $5.0 \pm 0.0$ & $6.0 \pm 0.7$ & $p=0.0001$ & $\mathrm{~ns}^{1}$ & ns \\
\hline \multicolumn{2}{|c|}{ PN6 } & $8.9 \pm 0.6$ & $8.6 \pm 0.8$ & $4.7 \pm 0.3$ & $5.25 \pm 0.8$ & $p=0.0001$ & ns & ns \\
\hline \multicolumn{2}{|c|}{ Cannibalism } & $1.5 \pm 0.5$ & $2.9 \pm 1.0$ & $1.0 \pm 0.7$ & $0.8 \pm 0.3$ & $\mathrm{~ns}$ & ns & ns \\
\hline \multicolumn{9}{|c|}{ Body Weight (g) } \\
\hline \multirow{2}{*}{ PN1 } & Male & $4.17 \pm 0.12$ & $4.13 \pm 0.10$ & $4.23 \pm 0.15$ & $3.99 \pm 0.13$ & ns & ns & ns \\
\hline & Female & $3.87 \pm 0.12$ & $3.98 \pm 0.06$ & $3.98 \pm 0.06$ & $3.94 \pm 0.11$ & ns & $\mathrm{ns}$ & ns \\
\hline \multirow{2}{*}{ PN3 } & Male & $5.62 \pm 0.11$ & $5.42 \pm 0.15$ & $5.41 \pm 0.14$ & $5.12 \pm 0.15$ & ns & ns & ns \\
\hline & Female & $5.38 \pm 0.12$ & $5.16 \pm 0.20$ & $5.23 \pm 0.14$ & $4.91 \pm 0.14$ & ns & ns & ns \\
\hline \multirow{2}{*}{ PN6 } & Male & $8.54 \pm 0.21$ & $8.31 \pm 0.23$ & $8.66 \pm 0.38$ & $7.65 \pm 0.30$ & ns & $p=0.039$ & ns \\
\hline & Female & $8.17 \pm 0.21$ & $7.91 \pm 0.37$ & $8.29 \pm 5.14$ & $7.26 \pm 0.28$ & ns & $p=0.049$ & ns \\
\hline \multirow{2}{*}{ PN10 } & Male & $14.6 \pm 0.3$ & $14.1 \pm 0.4$ & $14.6 \pm 0.5$ & $13.1 \pm 0.5$ & ns & $p=0.037$ & ns \\
\hline & Female & $14.2 \pm 0.3$ & $13.6 \pm 0.6$ & $14.1 \pm 0.5$ & $12.7 \pm 0.5$ & ns & $p=0.040$ & ns \\
\hline \multirow{2}{*}{ PN14 } & Male & $22.1 \pm 0.5$ & $21.2 \pm 0.6$ & $21.5 \pm 0.7$ & $19.6 \pm 1.0$ & ns & $p=0.043$ & ns \\
\hline & Female & $21.7 \pm 0.4$ & $20.6 \pm 0.8$ & $20.6 \pm 0.8$ & $19.0 \pm 0.9$ & $\mathrm{~ns}$ & $\mathrm{~ns}$ & ns \\
\hline \multirow{2}{*}{ PN17 } & Male & $27.4 \pm 0.6$ & $26.3 \pm 0.6$ & $26.9 \pm 0.8$ & $24.5 \pm 1.2$ & ns & $p=0.030$ & ns \\
\hline & Female & $26.9 \pm 0.5$ & $25.6 \pm 0.7$ & $26.0 \pm 0.8$ & $24.1 \pm 1.1$ & ns & $p=0.047$ & ns \\
\hline \multirow{2}{*}{ PN21 } & Male & $34.3 \pm 0.6$ & $33.6 \pm 1.0$ & $34.7 \pm 0.7$ & $31.4 \pm 1.1$ & ns & $p=0.020$ & ns \\
\hline & Female & $33.8 \pm 0.6$ & $32.8 \pm 1.0$ & $33.5 \pm 0.9$ & $31.4 \pm 1.0$ & ns & ns & ns \\
\hline \multirow{2}{*}{ PN24 } & Male & $43.7 \pm 1.1$ & $42.3 \pm 0.9$ & $44.3 \pm 1.0$ & $39.2 \pm 1.4$ & ns & $p=0.004$ & ns \\
\hline & Female & $42.1 \pm 0.8$ & $40.4 \pm 1.1$ & $42.4 \pm 1.1$ & $38.5 \pm 1.2$ & ns & $p=0.010$ & ns \\
\hline \multirow{2}{*}{ PN28 } & Male & $58.7 \pm 1.2$ & $57.4 \pm 1.1$ & $59.6 \pm 1.2$ & $54.0 \pm 1.8$ & ns & $p=0.011$ & ns \\
\hline & Female & $54.9 \pm 1.0$ & $54.1 \pm 1.4$ & $55.0 \pm 1.4$ & $51.3 \pm 1.3$ & ns & ns & ns \\
\hline \multirow{2}{*}{ PN35 } & Male & $87.7 \pm 1.7$ & $86.6 \pm 1.7$ & $87.1 \pm 1.3$ & $82.8 \pm 2.8$ & ns & ns & ns \\
\hline & Female & $79.1 \pm 1.2$ & $77.8 \pm 1.8$ & $77.0 \pm 1.4$ & $75.7 \pm 1.8$ & ns & ns & ns \\
\hline
\end{tabular}

Data are analysed with a two-way ANOVA reporting differences between Pup (Standard and Reduced litters) and Dam (Normal and Altered milk quality/composition) groups. Data presented as the mean $\pm \mathrm{SEM}$, where ns is not significant. ${ }^{1} \mathrm{~ns}$ is not significant.

\subsection{Mammary Structure and Maternal Plasma Analysis}

An interaction between Dam and Pup was identified in mammary weight, where it was decreased in Reduced litter size pups suckled by Normal milk quality/composition dams compared to Standard litter size pups suckled by Normal milk quality/composition dams (-40\%, Figure 2a; Tukey's post-hoc). There was, however, no difference in mammary weight between Standard litter size pups suckled by Altered or Normal milk quality/composition dams; likely due to an increased alveolar number $(+25 \%)$ in Altered milk quality/composition dams, but not area (Table 2$)$. There were no differences in maternal plasma PTHrP and total calcium between groups (Table 2). An interaction between Dam and Pup was identified in maternal ionic calcium, whereby it was increased in Reduced litter size pups suckled by Normal milk quality/composition dams compared to Standard litter size pups suckled by Normal milk quality/composition dams (+36\%) and Reduced litter size pups suckled by Altered milk quality/composition dams (+97\%), but was decreased in Reduced litter size pups suckled by 
Altered milk quality/composition dams compared to Standard litter size pups suckled by Altered milk quality /composition dams (-40\%; Figure 2b; Tukey's post-hoc). Maternal corticosterone (Table 2) along with mammary PTHrP (mRNA and protein; Table 2 and Figure 2c) were not affected by litter size or maternal treatment. A main Dam effect was identified in mammary a-lactalbumin gene abundance, where it was decreased in Altered milk quality/composition dams (-51\%; Figure 2d). An interaction between Dam and Pup was identified in mammary b-casein, which was lower in Standard litter size pups suckled by Altered milk quality/composition dams (-65\%) and Reduced litter size pups suckled by Normal milk quality/composition dams (-59\%) compared to Standard litter size pups suckled by Normal milk quality/composition dams (Figure 2e; Tukey's post-hoc).

\subsection{Pup Plasma Analysis and Milk Consumption}

Milk PTHrP tended to be increased in dams suckling Reduced litter size pups (Figure 3a; $p=0.060$, two-way ANOVA). Milk $\mathrm{Na}^{+} / \mathrm{K}^{+}$, ionic and total calcium, total protein and lactose were not affected by maternal milk quality/composition or litter size (Table 2). A main Pup effect was identified in pup plasma PTHrP, where it was increased in Reduced litter size pups (+69\%; Figure 3b). This finding was in conjunction with main Pup effects in pup ionic calcium and total body calcium, where they were decreased in Reduced litter size pups ( $-35 \%$ and $-5 \%$, respectively; Figure $3 c, d)$. Milk intake in both male and female pups were not affected by maternal milk quality/composition or pup litter size (Figure 3e,f).

Table 2. Maternal plasma, mammary and pooled milk composition in the four cross-foster groups on postnatal day 6.

\begin{tabular}{|c|c|c|c|c|c|c|c|}
\hline \multirow{2}{*}{ Pup-on-Dam } & \multirow{2}{*}{$\begin{array}{l}\text { Standard- } \\
\text { on-Normal }\end{array}$} & \multirow{2}{*}{$\begin{array}{l}\text { Standard- } \\
\text { on-Altered }\end{array}$} & \multirow{2}{*}{$\begin{array}{l}\text { Reduced- } \\
\text { on-Normal }\end{array}$} & \multirow{2}{*}{$\begin{array}{l}\text { Reduced- } \\
\text { on-Altered }\end{array}$} & \multicolumn{3}{|c|}{ Two-Way ANOVA } \\
\hline & & & & & Pup & Dam & Interaction \\
\hline \multicolumn{8}{|l|}{ Maternal ( $n=4-9$ per group) } \\
\hline Plasma total calcium (mmol/L) & $2.6 \pm 0.08$ & $2.6 \pm 0.04$ & $2.6 \pm 0.05$ & $2.4 \pm 0.04$ & ns & ns & ns \\
\hline Plasma corticosterone $(\mathrm{ng} / \mathrm{mL})$ & $593 \pm 59$ & $726 \pm 106$ & $551 \pm 86$ & $553 \pm 80$ & ns & ns & ns \\
\hline \multicolumn{8}{|l|}{ Mammary ( $n=3-6$ per group) } \\
\hline PTHrP mRNA & $1.0 \pm 0.1$ & $0.8 \pm 0.3$ & $1.1 \pm 0.2$ & $1.4 \pm 0.3$ & ns & ns & ns \\
\hline \multicolumn{8}{|l|}{ Milk ( $n=4-8$ per group) } \\
\hline $\mathrm{Na} / \mathrm{K}$ & $7.6 \pm 1.2$ & $6.5 \pm 1.6$ & $9.8 \pm 2.5$ & $6.4 \pm 0.9$ & ns & ns & ns \\
\hline Ionic calcium (mmol/L) & $8.9 \pm 0.6$ & $8.7 \pm 1.0$ & $7.4 \pm 0.9$ & $9.1 \pm 0.4$ & ns & ns & ns \\
\hline Total calcium (mmol/L) & $64.7 \pm 4.0$ & $65.8 \pm 3.7$ & $60.4 \pm 5.6$ & $57.9 \pm 2.8$ & ns & ns & ns \\
\hline
\end{tabular}

Data are analysed with a two-way ANOVA reporting differences between Pup (Standard and Reduced litters) and Dam (Normal and Altered milk quality/composition) groups. Data presented as the mean \pm SEM with sex pooled per litter, where ns is not significant. ${ }^{1} \mathrm{~ns}$ is not significant.

\subsection{Milk Fatty Acid Composition}

An interaction between Dam and Pup was identified in linoleic acid (LA), arachidonic acid (AA) and total n-6 fatty acids. Specifically, levels of the individual n-6 PUFAs (LA and AA), and total n-6 fatty acid content were increased in milk from Standard litter size pups suckled by Altered milk quality / composition dams ( $+20 \%$ and $+21 \%$ for LA and total $n-6)$ and Reduced litter size pups suckled by Normal milk quality/composition( $+21 \%,+48 \%$ and $+24 \%$, respectively) dams compared to Standard litter size pups suckled by Normal milk quality/composition dams (Table 3; Tukey's post-hoc). There was a main Pup effect in levels of the n-3 LC-PUFA docosahexaenoic acid (DHA) which was increased in milk from dams suckling Reduced litter size pups (+31\%; Table 3), but there was no difference in total n-3 fatty acid content of the milk between groups. An interaction between Dam and Pup was identified in long chain saturated fatty acid content, where they were decreased in the milk of Reduced litter size pups suckled by Normal milk quality/composition dams compared to Standard litter size pups suckled by Normal milk quality/composition dams (-24\%; Table 3, Tukey's 
post-hoc). A main Pup effect was identified in total LC-MUFA content, where it was increased in the milk of dams suckling Reduced litter pups (+11\%). The ratio of n-6:n-3 fatty acids and medium saturated fatty acids were not affected by litter size or maternal milk quality/composition (Table 3). The full list of fatty acids analyzed from the stomach contents are reported in Supplementary Table S1.

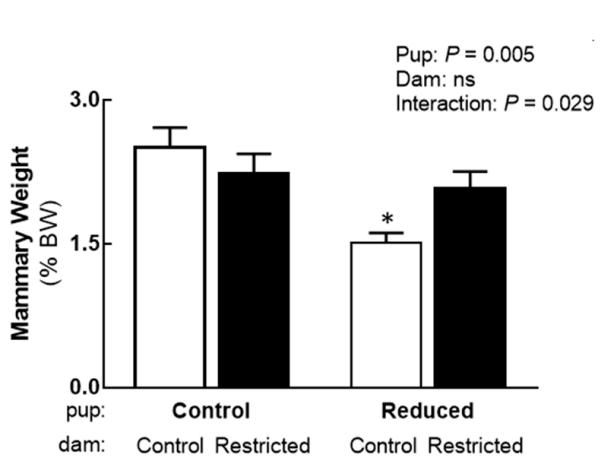

(a)

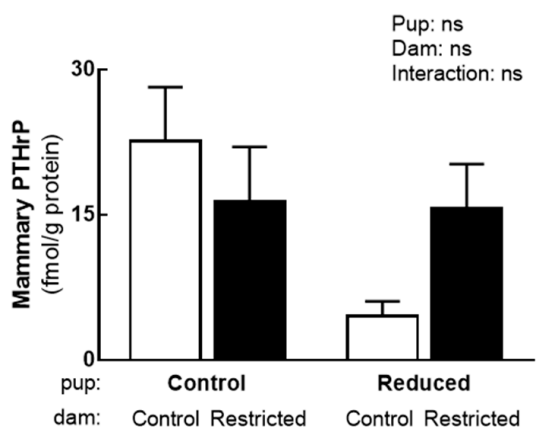

(c)

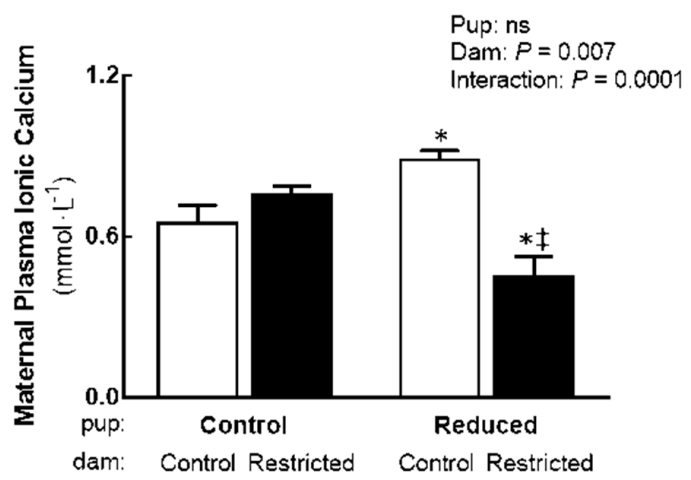

(b)

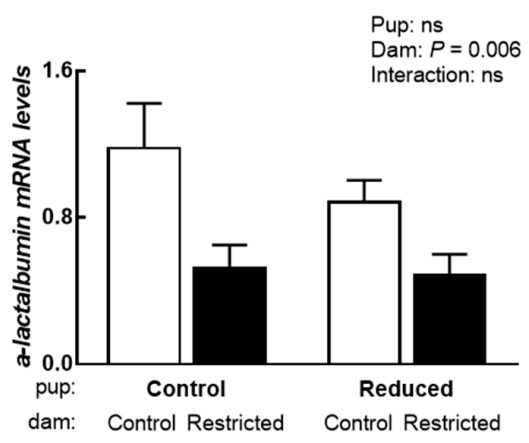

(d)

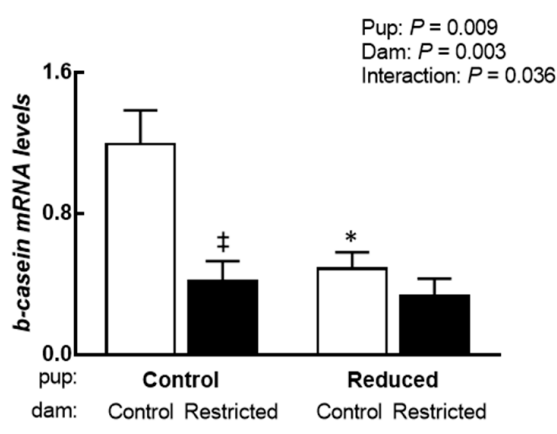

(e)

Figure 2. Effect reducing litter size has on maternal and mammary outcomes ( $n=5-9$ per group). (a) Mammary weight, (b) maternal ionic calcium concentrations and (c) mammary PTHrP protein concentration, (d) a-lactalbumin and (e) b-casein mRNA expression. Data are analysed with a two-way ANOVA reporting differences between Pup (Standard and Reduced litters) and Dam (Normal and Altered milk quality/composition) groups, with a Tukey's post-hoc test used to identify where interactions lie. Data presented as the mean \pm SEM, where ns is not significant. Significant differences between Standard and Reduced litter pups are indicated by an asterisk $\left({ }^{*} p<0.05\right)$ and differences between sham operated (Control; Normal milk quality/composition) and uteroplacental insufficiency surgery (Restricted; altered milk quality/composition) dams are indicated with a double dagger ( $p<0.05)$. Normal milk quality/composition dams denoted by white open bars and Altered milk quality/composition dams denoted by black closed bars. 


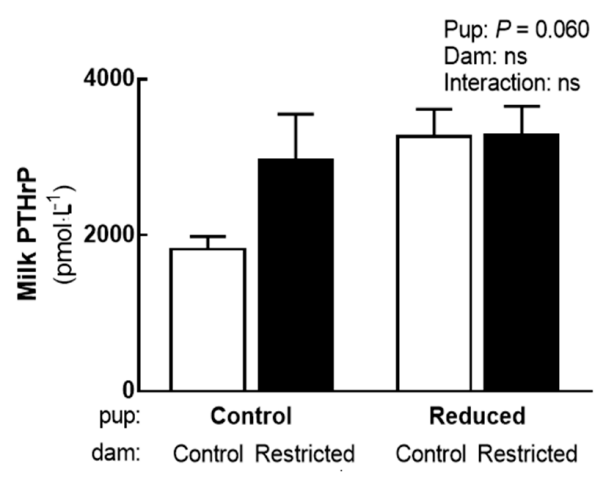

(a)

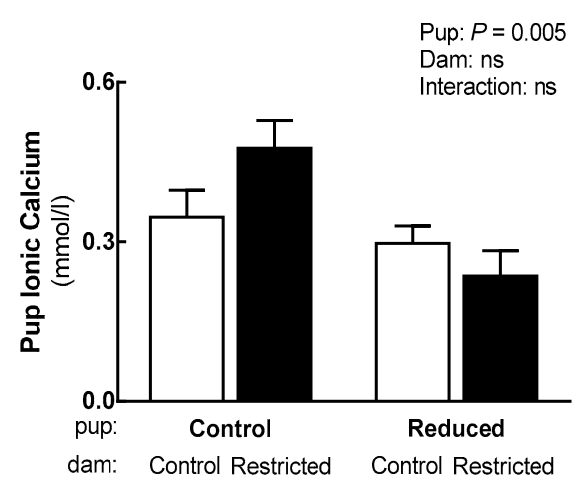

(c)

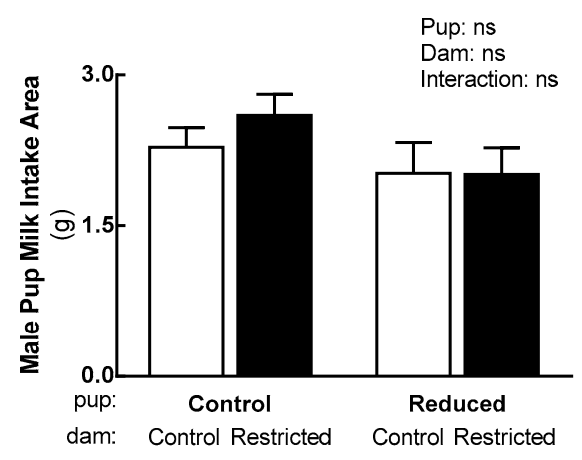

(e)

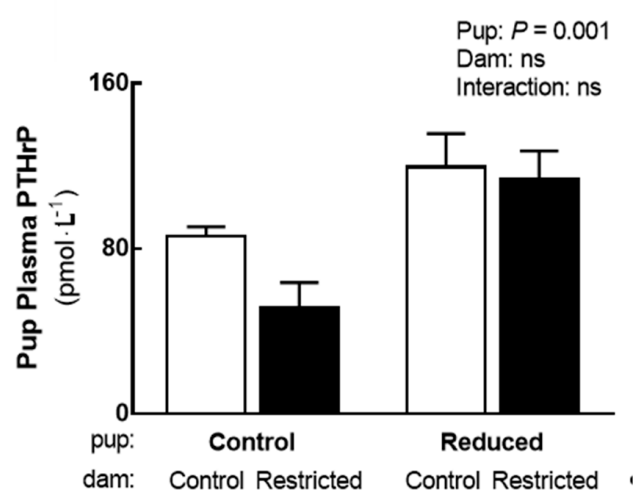

(b)

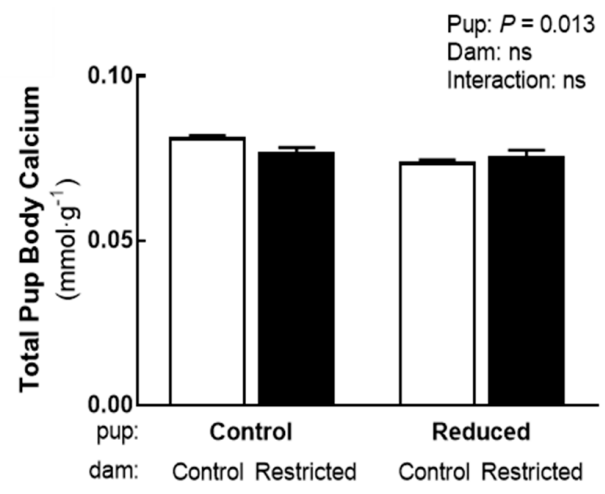

(d)

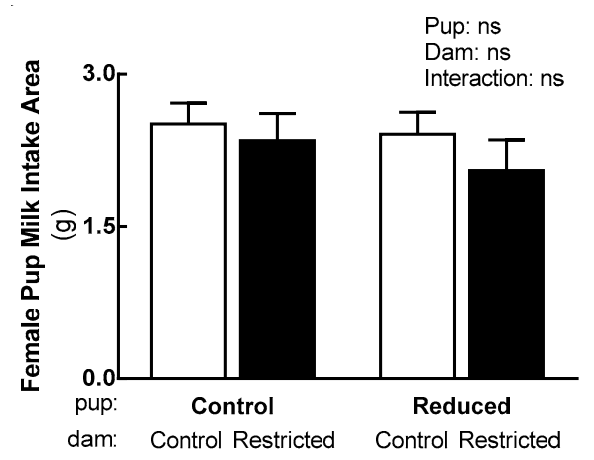

(f)

Figure 3. Effect reducing litter size has on pup calcium handling and milk intake $(n=6-10$ litter averages per group, where appropriate). (a) Milk and (b) pup PTHrP concentrations, (c) pup ionic calcium concentrations, (d) pup total body calcium and $(\mathbf{e}, \mathbf{f})$ pup milk intake. Data are analysed with a two-way ANOVA reporting differences between Pup (Standard and Reduced litters) and Dam (Normal and Altered milk quality/composition) groups. Data presented as the mean $\pm \mathrm{SEM}$, where ns is not significant. Normal milk quality/composition dams denoted by white open bars and Altered milk quality/composition dams denoted by black closed bars.

\subsection{Adult Health}

Body weight at 6 months was decreased in male $(-5 \%)$, but not female, offspring from Reduced litters, which was further exaggerated by Altered milk quality/composition $(-5 \%)$ (Table 4). Femur length in both males and females was unaffected by litter size or maternal milk quality/composition (Table 4). An interaction between Pup and Dam was identified in male and 
female trabecular mineral content, whereby trabecular mineral content was decreased in male $(-14 \%)$ and female $(-12 \%)$ Reduced litter size pups suckled by Altered milk quality/composition dams compared to Reduced litter size pups suckled by Normal milk quality/composition counterparts (Figure 4a; Tukey's post-hoc). A main Dam effect was identified in trabecular density in male offspring, where it decreased in offspring suckled by Altered milk quality/composition dams ( $-6 \%$; Figure $4 \mathrm{~b})$. In females, an interaction between Pup and Dam was identified trabecular density, where it was decreased in Reduced litter size pups suckled by Altered milk quality/composition dams $(-8 \%)$ compared to Reduced litter size pups suckled by Normal milk quality/composition dams (Figure 4b; Tukey's post-hoc). Cortical mineral content was decreased in male offspring who were suckled by Altered milk quality/composition dams $(-4 \%)$, with no change in females (Table 4$)$. Cortical density was unaffected by litter size or maternal milk quality/composition in both male and female offspring (Table 4). A main Pup effect was identified in bone bending strength in male, but not female offspring, where it was decreased in offspring of Reduced litters ( $-7 \%$; Figure $4 \mathrm{c})$.

Table 3. Fatty acid composition in stomach contents consumed in milk presented as a cumulative total percentage of fatty acids on postnatal day 6 in the four cross-foster groups $(n=5-7$ per group, with $n=1$ representing data from 1 pooled litter).

\begin{tabular}{|c|c|c|c|c|c|c|c|}
\hline \multirow[b]{2}{*}{ Pup-on-Dam } & \multirow{2}{*}{$\begin{array}{l}\text { Standard- } \\
\text { on-Normal }\end{array}$} & \multirow{2}{*}{$\begin{array}{l}\text { Standard- } \\
\text { on-Altered }\end{array}$} & \multirow{2}{*}{$\begin{array}{c}\text { Reduced- } \\
\text { on-Normal }\end{array}$} & \multirow{2}{*}{$\begin{array}{c}\text { Reduced- } \\
\text { on-Altered }\end{array}$} & \multicolumn{3}{|c|}{ Two-Way ANOVA } \\
\hline & & & & & Pup & Dam & Interaction \\
\hline \multicolumn{8}{|l|}{ Fatty Acids } \\
\hline \multicolumn{8}{|l|}{ Omega-6 PUFA } \\
\hline Linoleic $(18: 2 n-6)$ & $11.79 \pm 0.25$ & $14.12 \pm 0.43^{\ddagger 1}$ & $14.23 \pm 0.26 * 2$ & $13.85 \pm 0.50$ & $p=0.010$ & $p=0.019$ & $p=0.002$ \\
\hline Arachidonic (20:4n-6) & $1.03 \pm 0.06$ & $1.33 \pm 0.12$ & $1.52 \pm 0.08$ * & $1.35 \pm 0.09$ & $p=0.014$ & $\mathrm{~ns}^{3}$ & $p=0.023$ \\
\hline Total n-6 PUFA & $14.4 \pm 0.4$ & $17.4 \pm 0.7^{\ddagger}$ & $17.9 \pm 0.4^{*}$ & $17.1 \pm 0.7$ & $p=0.009$ & ns & $p=0.003$ \\
\hline \multicolumn{8}{|l|}{ Omega-3 PUFA } \\
\hline$\alpha$-linolenic (18:3n-3) & $1.40 \pm 0.06$ & $1.48 \pm 0.09$ & $1.49 \pm 0.05$ & $1.46 \pm 0.11$ & ns & ns & ns \\
\hline Eicosapentaenoic (20:5n-3) & $0.21 \pm 0.01$ & $0.17 \pm 0.03$ & $0.21 \pm 0.02$ & $0.18 \pm 0.04$ & ns & ns & ns \\
\hline Docosahexaenoic (22:6n-3) & $0.41 \pm 0.03$ & $0.45 \pm 0.06$ & $0.55 \pm 0.04$ & $0.58 \pm 0.09$ & $p=0.047$ & ns & ns \\
\hline Total n-3 PUFA & $2.4 \pm 0.09$ & $2.4 \pm 0.18$ & $2.7 \pm 0.15$ & $2.7 \pm 0.21$ & ns & ns & ns \\
\hline$n-6: n-3$ & $6.07 \pm 0.15$ & $7.11 \pm 0.27$ & $6.58 \pm 0.28$ & $6.63 \pm 0.54$ & ns & ns & ns \\
\hline Total LC-MUFA & $22.4 \pm 0.9$ & $22.4 \pm 1.4$ & $25.8 \pm 0.9$ & $23.9 \pm 0.8$ & $p=0.032$ & ns & ns \\
\hline \multicolumn{8}{|l|}{ Saturated Fatty Acids } \\
\hline Medium (6-12) & $19.8 \pm 1.0$ & $21.1 \pm 0.7$ & $21.1 \pm 0.7$ & $20.8 \pm 0.9$ & ns & ns & ns \\
\hline Long (14-20) & $40.4 \pm 1.1$ & $35.2 \pm 2.1$ & $30.9 \pm 1.4^{*}$ & $34.2 \pm 1.9$ & $p=0.006$ & ns & $p=0.022$ \\
\hline
\end{tabular}

Data are analysed with a two-way ANOVA reporting differences between Pup (Standard and Reduced litters) and Dam (Normal and Altered milk quality/composition) groups, with a Tukey's post-hoc test used to identify where interactions lie. Data presented as the mean \pm SEM with sex pooled per litter, where ns is not significant. Significant differences between Standard and Reduced litter pups are indicated by an asterisk $\left({ }^{*} p<0.05\right)$ and differences between sham operated (Control; Normal milk quality/composition) and uteroplacental insufficiency surgery (Restricted; Altered milk quality/composition) dams are indicated with a double dagger $\left({ }^{\ddagger} p<0.05\right)$. Also see Table S1 for individual fatty acids. ${ }^{1} p<0.05$ Standard-on-Altered vs. Standard-on-Normal; ${ }^{2} p<0.05$ Reduced-on-Normal vs. Standard-on-Normal; ${ }^{3}$ ns is not significant.
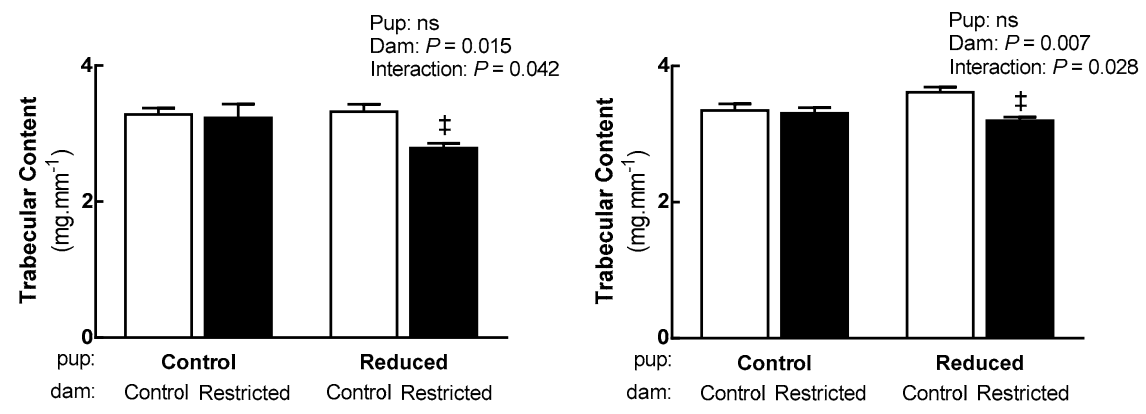

(a)

Figure 4. Cont. 

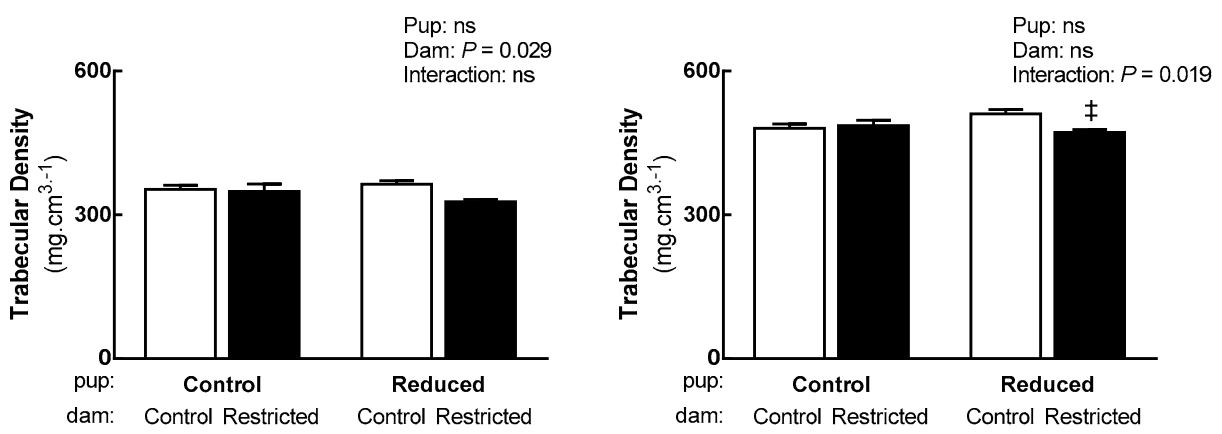

(b)
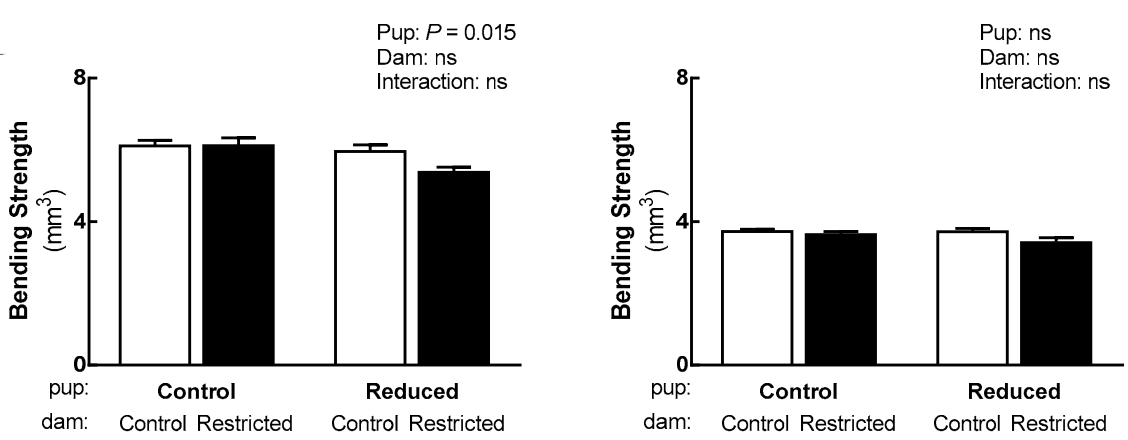

(c)
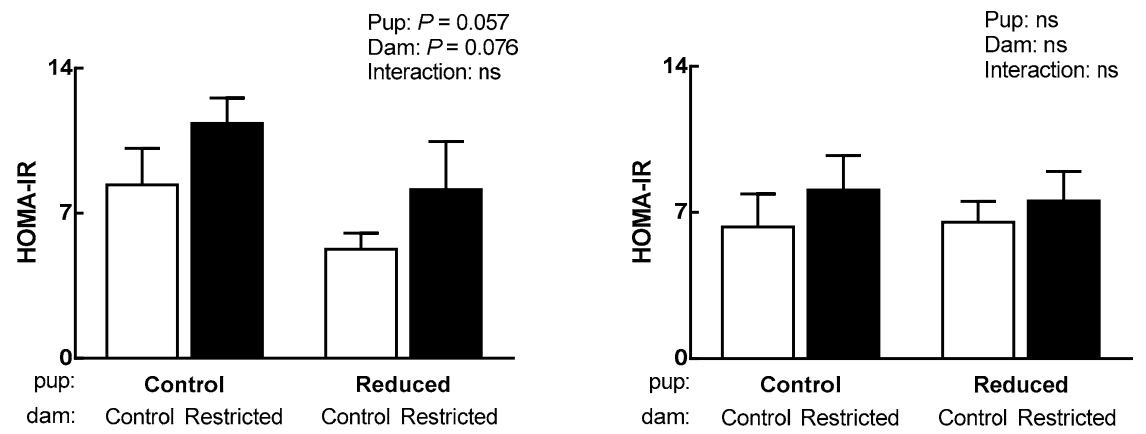

(d)
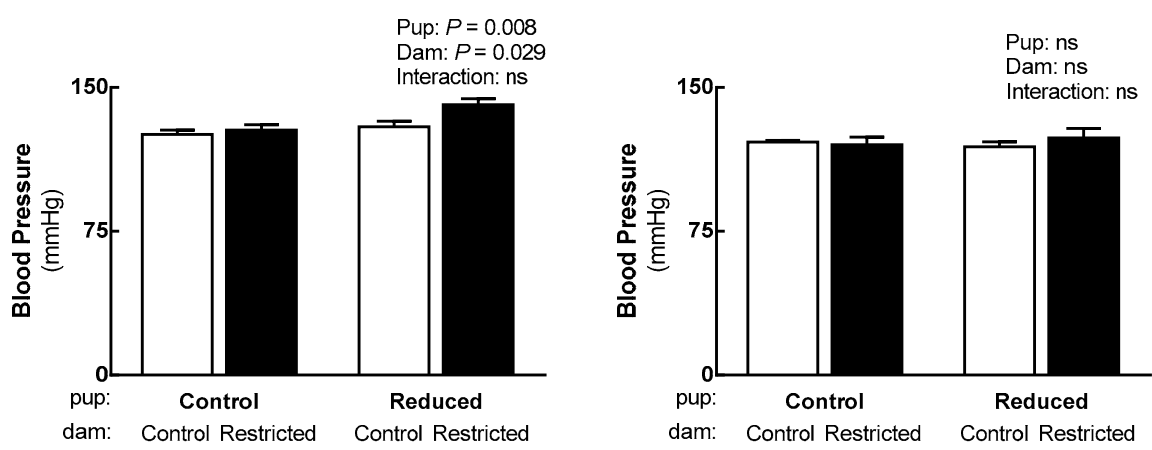

(e)

Figure 4. Effect reducing litter size has on adult offspring bone ( $n=9-19$ per group, with $n=1$ representing 1 male and female per litter) and cardiometabolic health ( $n=5-11$ per group, with $n=1$ 
representing 1 male and female per litter). (a,b) Trabecular mineral content and density, (c) bone bending strength, (d) homeostasis model assessment for insulin resistance (HOMA-IR) and (e) blood pressure at 6 months. Data are analysed with a two-way ANOVA reporting differences between Pup (Standard and Reduced litters) and Dam (Normal and Altered milk quality/composition) groups, with a Tukey's post-hoc test used to identify where interactions lie. Data presented as the mean \pm SEM, where ns is not significant. Significant differences between sham operated (Control; Normal milk quality/composition) and uteroplacental insufficiency surgery (Restricted; Altered milk quality/composition) dams are indicated with a double dagger $(\ddagger p<0.05)$. Normal milk quality/composition dams denoted by white open bars and Altered milk quality/composition dams denoted by black closed bars, with data from males on the left-hand side and data from females on the right-hand side.

Fasting glucose at 6 months was not different between groups in either males or females (Table 4). In male, but not female offspring, a main Dam effect was identified in fasting insulin concentrations where it was higher in offspring who had been suckled by Altered milk quality/composition dams and also tended to be lower in Reduced litter size pups (Table $4 ; p=0.070$ ). Similarly, glucose AUC tended to be higher and first phase insulin tended to be lower in male offspring that were suckling Altered milk quality / composition dams (Table 4; $p=0.080$ and $p=0.069$, respectively), with no changes in females. HOMA-IR at 6 months tended to be increased in offspring who had been suckled by Altered milk quality/composition dams $(p=0.076)$ and decreased in Reduced litter size offspring ( $p=0.057$; Figure 4d). Interestingly, a main Pup and Dam effect were observed in male blood pressure, where it was increased both in Reduced litter male offspring compared to Standard litter size offspring $(+7 \%)$ and in offspring who had been suckled by Altered milk quality/composition dams compared to Normal milk quality/composition dams (+5\%; Figure 4e). HOMA-IR and blood pressure were not different between groups in female offspring (Figure $4 \mathrm{~d}, \mathrm{e}$ ).

Table 4. Adult offspring physiology in the four cross-foster groups at 6 months $(n=9-19$ per group for bone analyses and $n=5-11$ per group for cardiometabolic analyses, with $n=1$ representing 1 male and female per litter).

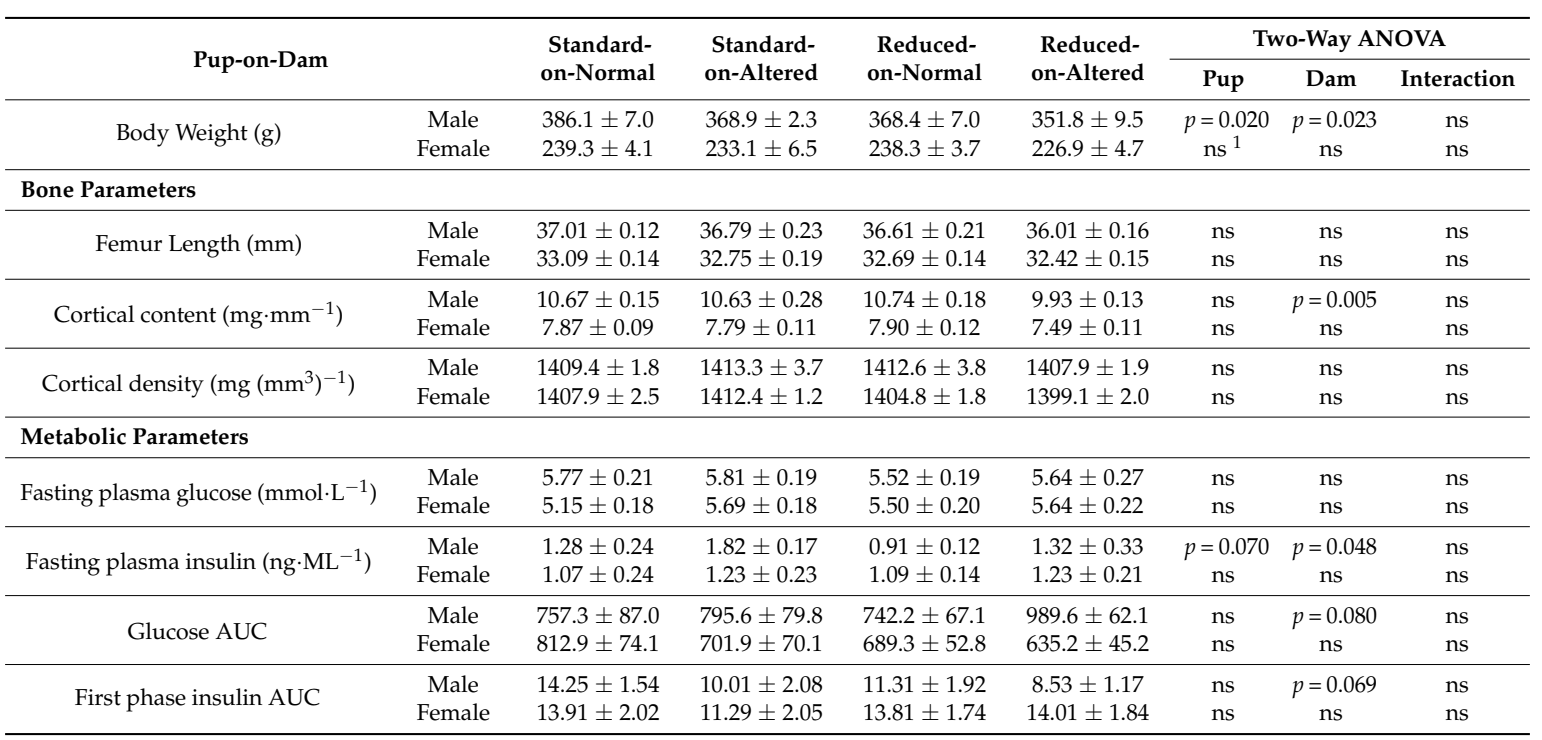

Data are analysed with a two-way ANOVA reporting differences between Pup (Standard and Reduced litters) and Dam (Normal and Altered milk quality/composition) groups. Data presented as the mean \pm SEM, where ns is not significant. ${ }^{1} \mathrm{~ns}$ is not significant.

\section{Discussion}

Providing offspring with adequate and appropriate nutrition during lactation is essential for long-term health, with both over- and undernutrition increasing disease susceptibility [14-16]. We have 
previously demonstrated that both the dam and suckling pup can influence maternal milk composition, and that these effects generally represent an attempt to improve pup growth and development [34]. The current study has demonstrated that reducing litter size at birth, regardless of whether the dam has normal or altered milk quality/composition impairs pup calcium homeostasis, which may reduce bone bending strength in male offspring. Additionally, these adult Reduced litter males appear to have increased cardiovascular disease risk and decreased diabetes risk. Furthermore, altered milk quality/composition (induced by uteroplacental insufficiency surgery) similarly programs poor bone health, high blood pressure and impairs glucose tolerance/insulin sensitivity. These finding are likely due to differential influences of the predetermined litter size at birth [26] and effects on milk quality. This highlights that caution needs to be taken when interpreting animal studies that focus on early life nutrition, as the experimental approach may independently modulate milk nutrition and quality.

\subsection{Effects of Reducing Litter Size}

It is important to note that as all dams underwent a surgical procedure (sham or ligation) and all litters were cross-fostered the effects reported are as direct consequences of either the maternal surgery (resulting in altered milk quality/composition) or reduced litter size, thus we have adequately accounted for all possible confounders, such as stress, across experimental groups. Additionally, growth restricted pups were not included in the study, only pups born from sham-operated dams that have intact or reduced litter sizes when cross-fostered, which would limit any changes observed in pups suckled by dams that underwent uteroplacental insufficiency surgery to the lactation environment they are exposed to in early postnatal life. Reducing litter size is commonly used in the developmental programming field, however recent studies demonstrate that this can independently program disease susceptibility [27-30]. In the current study we report dynamic changes in pup calcium homeostasis and maternal milk composition as a result of reducing the litter size to 5 pups. Interestingly, despite these changes in milk composition, no changes in postnatal body weight was observed, which suggests that other intrinsic hormonal factors may be responsible for maintaining pup growth that requires further studies. The reduction in mammary gland weight in Normal milk quality/composition dams suckled by Reduced litter pups may be due to the decreased $b$-casein gene expression, which is known to regulate mammary differentiation and is a regulator of milk protein gene expression [49]. However, this likely did not alter alveolar area or number as lactogenic differentiation is completed by PN6 [49]; although studies at earlier postnatal ages may reveal altered mammary structure. Despite no changes in maternal PTHrP, dams suckled by Reduced litter pups had increased milk PTHrP. Previous studies have demonstrated that milk PTHrP is not absorbed by the intestines into the pups circulation [50], suggesting that the increased pup PTHrP concentrations we report are independent of milk PTHrP content. This increased pup PTHrP is likely to stimulate bone resorption, and thus calcium release, in an attempt to increase plasma calcium concentrations, as shown in a previous study [51], but has also been shown to result in decreased postnatal body calcium content [52]. Interestingly, the increased maternal ionic calcium concentrations in Normal milk quality/composition dams suckled by Reduced litter pups are likely an attempt to increase milk calcium concentrations to compensate for this deficit. This, however, did not translate to increased milk calcium content at the time point investigated in this study, highlighting that milk composition needs to be assessed at additional postnatal ages. Nevertheless, the increased milk PTHrP in dams suckling Reduced litter pups would facilitate increased intestinal calcium reabsorption to increase pup plasma calcium concentrations, preventing any further bone breakdown. These data may highlight why the Reduced litter size pups suckled by Normal milk quality/quantity dams do not exhibit the same deficits in adult bone mineral content and density that are observed in the Reduced litter size pups suckled by Altered milk quality / quantity. Despite this, however, Reduced litter male offspring have decreased bending strength, indicative of increased fracture risk and thus poor bone health, suggesting that any compensatory changes in milk composition were not sufficient to fully prevent bone deficits. 
In addition to alterations in pup calcium homeostasis in the Reduced litter group, we also report changes in milk fatty acid content, highlighting that Reducing litter size has profound effects on maternal milk quality. Interestingly, milk n-6 fatty acid content was increased in the Reduced litter size pups suckled by Normal milk quality/quantity dams. This is significant, since increased n-6 fatty acid intakes have been associated with increased fat deposition in early life [11]. High intakes of n-6 PUFA during lactation also influence lipid tissue status and modulate metabolic pathways that can lead to diabetes and cardiovascular disease [11]. It is important to note, however, that it is the balance of omega- 6 and omega-3 PUFA in the diet that appears to be the more important determinant of physiological effects than levels of either PUFA type alone. In the current study, the n-6:n-3 ratio was not altered, at least at this age, by either litter size or maternal uteroplacental insufficiency surgery. This suggests that the observed changes in milk PUFA composition may not be a major factor contributing to the male onset cardiovascular disease we report, but may instead be due to the decreased post-weaning growth trajectory. Interestingly, reducing litter size increased milk LC-MUFA and DHA (Reduced litter size pups suckled by Normal milk quality/quantity dams only) content, which is known to have beneficial effects on metabolic function during critical periods of development [11]. The improved metabolic health in Reduced litter size pups suckled by Normal milk quality/quantity dams male offspring may also be attributed to increased milk Pentadecanoic acid (15:0) and reduced Palmitic acid (16:0) both of which are known to reduce the risk of type 2 diabetes [53]. This may explain why the Reduced litter size male offspring appear to have a decreased risk of developing diabetes.

\subsection{Effects of Altered Milk Quality/Composition}

Surprisingly alterations in milk quality/composition, induced by maternal uteroplacental insufficiency surgery, resulted in very few changes in milk composition compared to the large number of changes associated with reducing litter size and slowing offspring postnatal growth. In the current study, we report that dams with altered milk quality/composition have decreased mammary a-lactalbumin gene abundance. If this translates to decreased milk $\alpha$-lactalbumin protein then this may, at least in part, contribute to the poor adult bone health we observed in male offspring reared by Altered milk quality/composition dams. Specifically, as $\alpha$-lactalbumin binds calcium [54] it is possible that decreased milk $\alpha$-lactalbumin impairs calcium delivery to the pup, compromising bone mineralization and development. This poor bone health was only apparent in Reduced litter size pups suckled by Altered milk quality/composition dams, likely because the effect was compounded by the decreased maternal ionic concentrations that may have compromised calcium delivery at later lactational ages. Interestingly, a recent study demonstrated that supplementing 6-week old obese diabetic Zucker rats with $\alpha$-lactalbumin for 13 weeks improves metabolic function [55]; highlighting the benefits of $\alpha$-lactalbumin on metabolic health. If the inverse is also true, and rats are exposed to decreased $\alpha$-lactalbumin during development, it is possible that this may contribute to the increased risk of adult metabolic disease we observed in our rats. In addition, the increased n- 6 fatty acid intake in Standard litter size pups suckled by Altered milk quality/quantity dams during lactation may also contribute to the increased blood pressure and impaired insulin sensitivity in male offspring [11]. As the female offspring suckled by Altered milk quality/composition dams caught up in body weight prior to weaning they may be protected against developing cardiometabolic disease, due to the benefits of early accelerated growth for long-term cardiometabolic health [36,56-58].

\subsection{Study Limitations}

Despite our study demonstrating that reducing the litter size from 9 pups to 5 pups alters milk quality/composition and has implications for long-term offspring health, further studies are required to investigate the effects of the more common practice of reducing litter sizes to 8-10 pups from original litter sizes of 12-20 pups, since this also has the potential to influence milk quality / quantity and thus offspring outcomes. Nevertheless, the findings of the current study highlight the potential impact 
reducing litter size, independent of other neonatal factors, has on programming offspring outcomes. An important factor that was not taken into consideration in the present study is the impact of offspring sex on milk composition, due to difficulties in controlling for this in our large litter bearing animal model. Indeed, several epidemiological and experimental (with smaller litter bearing animals) studies have well demonstrated differences in milk composition between male and female infants [59,60]; demonstrating that infant sex has a significant impact on milk quality. This highlights the need for well-controlled human and animal studies to identify alterations in milk composition between infant sexes in several different pregnancy complications.

A limitation of the current study is that maternal behavior was not evaluated throughout lactation. This is particularly important, as the quality and quantity of maternal care during lactation can impact on offspring behavioral, endocrine and neural development (see review by Curley and Champagne [61]). Specifically, a recent study demonstrated that dams whose litters were reduced to 3 pups (to induce postnatal overnutrition) have improved maternal care, characterize by increased time devoted to arched nursing and licking pups [62]. Not surprisingly the pups had early accelerated growth and were overweight by PN60, as indicated by increased adiposity, and were hyperglycemic and hyperleptinemic [62]. These findings are however difficult to compare directly to the results of the current study where the Reduced litter pups had decreased postnatal growth, which suggests that the extent of litter size reduction in the current study is unlikely to have resulted in improved maternal care.

\section{Conclusions}

This study demonstrates that reducing pup litter size and altered maternal milk quality/composition differentially program poor adult offspring health, which is likely due to altered milk quality. Specifically, reducing the litter size alters milk composition, impairs pup calcium homeostasis and programs poor bone health, which likely contributes to the lower diabetes risk and poor cardiovascular health. Alterations in maternal milk quality/composition, on the other hand, programs poor adult bone and cardiovascular health and reduces glucose tolerance/insulin sensitivity. This study highlights the need for appropriate controls in developmental research to clearly ascertain phenotypes in the model. Importantly, controls implemented to standardize outcomes across treatment groups may independently program disease susceptibility, which limit their translatability. Therefore, care must be taken in interpreting findings from studies that standardize litter size as it may mask subtle effects on cardiometabolic health.

Supplementary Materials: The following are available online at http:/ / www.mdpi.com/2072-6643/11/1/118/s1, Table S1: Fatty acid composition in stomach contents on postnatal day 6.

Author Contributions: Conceptualization, M.E.W. and K.M.M.; Methodology, M.E.W. and K.M.M.; Validation, J.F.B., R.O., T.R., B.S.M., K.M.M. and M.E.W.; Formal analysis, J.F.B., R.O., T.R., B.S.M., K.M.M. and M.E.W.; Investigation, J.F.B., R.O. and T.R.; Resources, M.E.W. and K.M.M; Data curation, J.F.B., R.O., T.R. and M.E.W.; Writing-original draft preparation, J.F.B.; Writing—review and editing, R.O., T.R., B.S.M., K.M.M. and M.E.W.; Visualization, J.F.B., B.S.M. and M.E.W.; Supervision, M.E.W. and K.M.M.; Project administration, M.E.W.; Funding acquisition, M.E.W. and K.M.M.

Funding: This research was funded by the National Health and Medical Research Council (NHMRC) of Australia, grant numbers 208948 (M.E.W.) and 400003 (M.E.W. and K.M.M.). J.F.B. holds a Faculty of Medicine, Dentistry and Health Sciences Postdoctoral Fellowship at the University of Melbourne. K.M.M. was funded by a NHMRC Senior Research Fellowship.

Acknowledgments: We thank Ursula Lorenc for her technical assistance and Jane Moseley for her assistance in this project. We also thank Andrew Sinclair for his assistance with the fatty acid analysis, Kevin Nicholas for his assistance with mammary gland morphology, and Andrew Jefferies and Kerryn Westcott for their assistance with the animal work involved in this project.

Conflicts of Interest: The authors declare no conflict of interest. The funders had no role in the design of the study; in the collection, analyses, or interpretation of data; in the writing of the manuscript, or in the decision to publish the results. 


\section{References}

1. Barker, D.J.; Winter, P.D.; Osmond, C.; Margetts, B.; Simmonds, S.J. Weight in infancy and death from ischaemic heart disease. Lancet 1989, 2, 577-580. [CrossRef]

2. Barker, D.J.P.; Godfrey, K.M.; Osmond, C.; Bull, A. The relation of fetal length, ponderal index and head circumference to blood pressure and the risk of hypertension in adult life. Paediatr. Perinat. Epidemiol. 1992, 6, 35-44. [CrossRef] [PubMed]

3. Gluckman, P.D.; Harding, J.E. Fetal growth retardation: Underlying endocrine mechanisms and postnatal consequences. Acta Paediatr. Scand. 1997, 422, 69-72. [CrossRef]

4. Wlodek, M.E.; Owens, J.A.; Siebel, A.L.; Moritz, K. Reduced nephron endowment and hypertension emerge following placental restriction in the rat. Pediatr. Res. 2005, 58, 1024.

5. Painter, R.C.; Roseboom, T.J.; Bleker, O.P. Prenatal exposure to the dutch famine and disease in later life: An overview. Reprod. Toxicol. 2005, 20,345-352. [CrossRef]

6. Jaquiery, A.L.; Oliver, M.H.; Honeyfield-Ross, M.; Harding, J.E.; Bloomfield, F.H. Periconceptional undernutrition in sheep affects adult phenotype only in males. J. Nutr. Metab. 2012, 2012, 123610. [CrossRef]

7. Sokol, R.J.; Delaney-Black, V.; Nordstrom, B. Fetal alcohol spectrum disorder. JAMA 2003, 290, $2996-2999$. [CrossRef]

8. Gray, S.P.; Denton, K.M.; Cullen-McEwen, L.; Bertram, J.F.; Moritz, K.M. Prenatal exposure to alcohol reduces nephron number and raises blood pressure in progeny. J. Am. Soc. Nephrol. 2010, 21, 1891-1902. [CrossRef]

9. Virk, J.; Li, J.; Vestergaard, M.; Obel, C.; Kristensen, J.K.; Olsen, J. Prenatal exposure to bereavement and type-2 diabetes: A danish longitudinal population based study. PLoS ONE 2012, 7, e43508.

10. Singh, R.R.; Cuffe, J.S.; Moritz, K.M. Short- and long-term effects of exposure to natural and synthetic glucocorticoids during development. Clin. Exp. Pharmacol. Physiol. 2012, 39, 979-989. [CrossRef]

11. Mennitti, L.V.; Oliveira, J.L.; Morais, C.A.; Estadella, D.; Oyama, L.M.; Oller do Nascimento, C.M.; Pisani, L.P. Type of fatty acids in maternal diets during pregnancy and/or lactation and metabolic consequences of the offspring. J. Nutr. Biochem. 2015, 26, 99-111. [CrossRef] [PubMed]

12. Ailhaud, G.; Massiera, F.; Weill, P.; Legrand, P.; Alessandri, J.M.; Guesnet, P. Temporal changes in dietary fats: Role of n-6 polyunsaturated fatty acids in excessive adipose tissue development and relationship to obesity. Prog. Lipid Res. 2006, 45, 203-236. [CrossRef] [PubMed]

13. Gallo, L.A.; Tran, M.; Moritz, K.M.; Wlodek, M.E. Developmental programming: Variations in early growth and adult disease. Clin. Exp. Pharmacol. Physiol. 2013, 40, 795-802. [CrossRef] [PubMed]

14. Pomar, C.A.; van, N.R.; Sanchez, J.; Pico, C.; Keijer, J.; Palou, A. Maternal consumption of a cafeteria diet during lactation in rats leads the offspring to a thin-outside-fat-inside phenotype. Int. J. Obes. (Lond.) 2017, 41, 1279-1287. [CrossRef] [PubMed]

15. Lozano, G.; Elmaghrabi, A.; Salley, J.; Siddique, K.; Gattineni, J.; Baum, M. Effect of prenatal programming and postnatal rearing on glomerular filtration rate in adult rats. Am. J. Physiol. Ren. Physiol. 2015, 308, F411-F419. [CrossRef] [PubMed]

16. Siddique, K.; Guzman, G.L.; Gattineni, J.; Baum, M. Effect of postnatal maternal protein intake on prenatal programming of hypertension. Reprod. Sci. 2014, 21, 1499-1507. [CrossRef] [PubMed]

17. Dickinson, H.; Moss, T.J.; Gatford, K.L.; Moritz, K.M.; Akison, L.; Fullston, T.; Hryciw, D.H.; Maloney, C.A.; Morris, M.J.; Wooldridge, A.L.; et al. A review of fundamental principles for animal models of dohad research: An australian perspective. J. Dev. Orig. Health Dis. 2016, 7, 449-472. [CrossRef] [PubMed]

18. De Almeida, F.J.; Duque-Guimaraes, D.; Carpenter, A.A.; Loche, E.; Ozanne, S.E. A post-weaning obesogenic diet exacerbates the detrimental effects of maternal obesity on offspring insulin signaling in adipose tissue. Sci. Rep. 2017, 7, 44949. [CrossRef]

19. Galyon, K.D.; Farshidi, F.; Han, G.; Ross, M.G.; Desai, M.; Jellyman, J.K. Maternal bisphenol a exposure alters rat offspring hepatic and skeletal muscle insulin signaling protein abundance. Am. J. Obstet. Gynecol. 2017, 216, 290.e1-290.e9. [CrossRef]

20. Maniam, J.; Antoniadis, C.P.; Wang, K.W.; Morris, M.J. Early life stress induced by limited nesting material produces metabolic resilience in response to a high-fat and high-sugar diet in male rats. Front. Endocrinol. (Lausanne) 2015, 6, 138. [CrossRef]

21. Firth, E.C.; Gamble, G.D.; Cornish, J.; Vickers, M.H. Neonatal leptin treatment reverses the bone-suppressive effects of maternal undernutrition in adult rat offspring. Sci. Rep. 2017, 7, 7686. [CrossRef] [PubMed] 
22. Dasinger, J.H.; Intapad, S.; Backstrom, M.A.; Carter, A.J.; Alexander, B.T. Intrauterine growth restriction programs an accelerated age-related increase in cardiovascular risk in male offspring. Am. J. Physiol. Ren. Physiol. 2016, 311, F312-F319. [CrossRef] [PubMed]

23. Zambrano, E.; Sosa-Larios, T.; Calzada, L.; Ibanez, C.A.; Mendoza-Rodriguez, C.A.; Morales, A.; Morimoto, S. Decreased basal insulin secretion from pancreatic islets of pups in a rat model of maternal obesity. J. Endocrinol. 2016, 231, 49-57. [CrossRef] [PubMed]

24. Niu, Y.; Herrera, E.A.; Evans, R.D.; Giussani, D.A. Antioxidant treatment improves neonatal survival and prevents impaired cardiac function at adulthood following neonatal glucocorticoid therapy. J. Physiol. 2013, 591, 5083-5093. [CrossRef] [PubMed]

25. Shah, A.; Reyes, L.M.; Morton, J.S.; Fung, D.; Schneider, J.; Davidge, S.T. Effect of resveratrol on metabolic and cardiovascular function in male and female adult offspring exposed to prenatal hypoxia and a high-fat diet. J. Physiol. 2016, 594, 1465-1482. [CrossRef] [PubMed]

26. Chahoud, I.; Paumgartten, F.J. Influence of litter size on the postnatal growth of rat pups: Is there a rationale for litter-size standardization in toxicity studies? Environ. Res. 2009, 109, 1021-1027. [CrossRef] [PubMed]

27. Wlodek, M.E.; Westcott, K.; Siebel, A.L.; Owens, J.A.; Moritz, K.M. Growth restriction before or after birth reduces nephron number and increases blood pressure in male rats. Kidney Int. 2008, 74, 187-195. [CrossRef] [PubMed]

28. Tare, M.; Parkington, H.C.; Bubb, K.J.; Wlodek, M.E. Uteroplacental insufficiency and lactational environment separately influence arterial stiffness and vascular function in adult male rats. Hypertension 2012, 60, 378-386. [CrossRef]

29. Tran, M.; Young, M.E.; Jefferies, A.J.; Hryciw, D.H.; Ward, M.M.; Fletcher, E.L.; Wlodek, M.E.; Wadley, G.D. Uteroplacental insufficiency leads to hypertension, but not glucose intolerance or impaired skeletal muscle mitochondrial biogenesis, in 12-month-old rats. Physiol. Rep. 2015, 3, e12556. [CrossRef]

30. O’Dowd, R.; Kent, J.C.; Moseley, J.M.; Wlodek, M.E. Effects of uteroplacental insufficiency and reducing litter size on maternal mammary function and postnatal offspring growth. Am. J. Physiol. Regul. Integr. Comp. Physiol. 2008, 294, R539-R548. [CrossRef]

31. Romano, T.; Wark, J.D.; Owens, J.A.; Wlodek, M.E. Prenatal growth restriction and postnatal growth restriction followed by accelerated growth independently program reduced bone growth and strength. Bone 2009, 45, 132-141. [CrossRef] [PubMed]

32. Habbout, A.; Guenancia, C.; Lorin, J.; Rigal, E.; Fassot, C.; Rochette, L.; Vergely, C. Postnatal overfeeding causes early shifts in gene expression in the heart and long-term alterations in cardiometabolic and oxidative parameters. PLoS ONE 2013, 8, e56981. [CrossRef] [PubMed]

33. Habbout, A.; Delemasure, S.; Goirand, F.; Guilland, J.C.; Chabod, F.; Sediki, M.; Rochette, L.; Vergely, C. Postnatal overfeeding in rats leads to moderate overweight and to cardiometabolic and oxidative alterations in adulthood. Biochimie 2012, 94, 117-124. [CrossRef] [PubMed]

34. Briffa, J.F.; O’Dowd, R.; Moritz, K.M.; Romano, T.; Jedwab, L.R.; McAinch, A.J.; Hryciw, D.H.; Wlodek, M.E. Uteroplacental insufficiency reduces rat plasma leptin concentrations and alters placental leptin transporters: Ameliorated with enhanced milk intake and nutrition. J. Physiol. 2017, 595, 3389-3407. [CrossRef] [PubMed]

35. Wlodek, M.E.; Westcott, K.T.; O’Dowd, R.; Serruto, A.; Wassef, L.; Moritz, K.M.; Moseley, J.M. Uteroplacental restriction in the rat impairs fetal growth in association with alterations in placental growth factors including pthrp. Am. J. Physiol. Regul. Integr. Comp. Physiol. 2005, 288, R1620-R1627. [CrossRef] [PubMed]

36. Wlodek, M.E.; Mibus, A.; Tan, A.; Siebel, A.L.; Owens, J.A.; Moritz, K.M. Normal lactational environment restores nephron endowment and prevents hypertension after placental restriction in the rat. J. Am. Soc. Nephrol. 2007, 18, 1688-1696. [CrossRef] [PubMed]

37. Moritz, K.M.; Mazzuca, M.Q.; Siebel, A.L.; Mibus, A.; Arena, D.; Tare, M.; Owens, J.A.; Wlodek, M.E. Uteroplacental insufficiency causes a nephron deficit, modest renal insufficiency but no hypertension with ageing in female rats. J. Physiol. 2009, 587, 2635-2646. [CrossRef]

38. Siebel, A.L.; Mibus, A.; De Blasio, M.J.; Westcott, K.T.; Morris, M.J.; Prior, L.; Owens, J.A.; Wlodek, M.E. Improved lactational nutrition and postnatal growth ameliorates impairment of glucose tolerance by uteroplacental insufficiency in male rat offspring. Endocrinology 2008, 149, 3067-3076. [CrossRef]

39. Wlodek, M.E.; Westcott, K.T.; Ho, P.W.M.; Serruto, A.; Di Nicolantonio, R.; Farrugia, W.; Moseley, J.M. Reduced fetal, placental, and amniotic fluid pthrp in the growth-restricted spontaneously hypertensive rat. Am. J. Physiol. Regul. Integr. Comp. Physiol. 2000, 279, R31-R38. [CrossRef] 
40. Wlodek, M.E.; Westcott, K.T.; Serruto, A.; O’Dowd, R.; Wassef, L.; Ho, P.W.M.; Moseley, J.M. Impaired mammary function and parathyroid hormone-related protein during lactation in growth-restricted spontaneously hypertensive rats. J. Endocrinol. 2003, 177, 233-245. [CrossRef]

41. Wlodek, M.E.; Ho, P.W.M.; Rice, G.E.; Moseley, J.M.; Martin, T.J.; Brennecke, S.P. Parathyroid hormone-related protein (pthrp) concentrations in human amniotic fluid during gestation and at the time of labour. Reprod. Fertil. Dev. 1995, 7, 1509-1513. [CrossRef] [PubMed]

42. Wlodek, M.E.; Ceranic, V.; O’Dowd, R.; Westcott, K.T.; Siebel, A.L. Maternal progesterone treatment rescues the mammary impairment following uteroplacental insufficiency and improves postnatal pup growth in the rat. Reprod. Sci. 2009, 16, 380-390. [CrossRef]

43. LePage, G.; Roy, C.C. Direct transesterification of all lipid classes in a one-step reaction. J. Lipid Res. 1986, 27, 114-120. [PubMed]

44. Weisinger, H.S.; Vingrys, A.; Bui, B.; Sinclair, A.J. Effects of dietary n-3 fatty acid deficiency and repletion in the guinea pig retina. Investig. Ophthalmol. Vis. Sci. 1999, 40, 327-338.

45. Anevska, K.; Gallo, L.A.; Tran, M.; Jefferies, A.J.; Wark, J.D.; Wlodek, M.E.; Romano, T. Pregnant growth restricted female rats have bone gains during late gestation which contributes to second generation adolescent and adult offspring having normal bone health. Bone 2015, 74, 199-207. [CrossRef] [PubMed]

46. Romano, T.; Wark, J.D.; Wlodek, M.E. Developmental programming of bone deficits in growth-restricted offspring. Reprod. Fertil. Dev. 2014, 27, 823-833. [CrossRef]

47. Anevska, K.; Cheong, J.N.; Wark, J.D.; Wlodek, M.E.; Romano, T. Maternal stress does not exacerbate long-term bone deficits in female rats born growth restricted, with differential effects on offspring bone health. Am. J. Physiol. Regul. Integr. Comp. Physiol. 2018, 314, R161-R170. [CrossRef]

48. Wadley, G.D.; Siebel, A.L.; Cooney, G.J.; McConell, G.K.; Wlodek, M.E.; Owens, J.A. Uteroplacental insufficiency and reducing litter size alters skeletal muscle mitochondrial biogenesis in a sex specific manner in the adult rat. Am. J. Physiol. Endocrinol. Metab. 2008, 294, E861-E869. [CrossRef]

49. Rijnkels, M.; Kabotyanski, E.; Montazer-Torbati, M.B.; Hue, B.C.; Vassetzky, Y.; Rosen, J.M.; Devinoy, E. The epigenetic landscape of mammary gland development and functional differentiation. J. Mammary Gland Biol. Neoplasia 2010, 15, 85-100. [CrossRef]

50. Kukreja, S.C.; D’Anza, J.J.; Melton, M.E.; Wimbiscus, S.A.; Grill, V.; Martin, T.J. Lack of effects of neutralisation of parathyroid hormone-related protein on calcium homeostasis in neonatal mice. J. Bone Miner. Res. 1991, 6 , 1197-1201. [CrossRef]

51. Kovacs, C.S. Calcium metabolism during pregnancy and lactation. J. Mammary Gland Biol. Neoplasia 2005, 10, 105-118. [CrossRef] [PubMed]

52. Kovacs, C.S. Bone development and mineral homeostasis in the fetus and neonate: Roles of the calciotropic and phosphotropic hormones. Physiol. Rev. 2014, 94, 1143-1218. [CrossRef] [PubMed]

53. Hirahatake, K.M.; Slavin, J.L.; Maki, K.C.; Adams, S.H. Associations between dairy foods, diabetes, and metabolic health: Potential mechanisms and future directions. Metabolism 2014, 63, 618-627. [CrossRef]

54. Permyakov, E.A.; Berliner, L.J. Alpha-lactalbumin: Structure and function. FEBS Lett. 2000, 473, $269-274$. [CrossRef]

55. Gregersen, S.; Bystrup, S.; Overgaard, A.; Jeppesen, P.B.; Sonderstgaard Thorup, A.C.; Jensen, E.; Hermansen, K. Effects of whey proteins on glucose metabolism in normal wistar rats and zucker diabetic fatty (zdf) rats. Rev. Diabet. Stud. 2013, 10, 252-269. [CrossRef] [PubMed]

56. Eriksson, J.; Forsen, T.; Tuomilehto, J.; Osmond, C.; Barker, D. Fetal and childhood growth and hypertension in adult life. Hypertension 2000, 36, 790-794. [CrossRef]

57. Lucas, A.; Fewtrell, M.S.; Davies, P.S.; Bishop, N.J.; Clough, H.; Cole, T.J. Breastfeeding and catch-up growth in infants born small for gestational age. Acta Paediatr. 1997, 86, 564-569. [CrossRef]

58. Eriksson, J.G.; Forsén, T.; Tuomilehto, J.; Osmond, C.; Barker, D.J.P. Early growth and coronary heart disease in later life: Longitudinal study. BMJ 2001, 322, 948-953. [CrossRef]

59. Galante, L.; Milan, A.M.; Reynolds, C.M.; Cameron-Smith, D.; Vickers, M.H.; Pundir, S. Sex-specific human milk composition: The role of infant sex in determining early life nutrition. Nutrients 2018, 10, 1194. [CrossRef]

60. Thakkar, S.K.; Giuffrida, F.; Cristina, C.H.; De Castro, C.A.; Mukherjee, R.; Tran, L.A.; Steenhout, P.; Lee le, Y.; Destaillats, F. Dynamics of human milk nutrient composition of women from singapore with a special focus on lipids. Am. J. Hum. Biol. 2013, 25, 770-779. [CrossRef] 
61. Curley, J.P.; Champagne, F.A. Influence of maternal care on the developing brain: Mechanisms, temporal dynamics and sensitive periods. Front. Neuroendocrinol. 2016, 40, 52-66. [CrossRef] [PubMed]

62. Enes-Marques, S.; Giusti-Paiva, A. Litter size reduction accentuates maternal care and alters behavioral and physiological phenotypes in rat adult offspring. J. Physiol. Sci. 2018, 68, 789-798. [CrossRef] [PubMed]

(c) 2019 by the authors. Licensee MDPI, Basel, Switzerland. This article is an open access article distributed under the terms and conditions of the Creative Commons Attribution (CC BY) license (http://creativecommons.org/licenses/by/4.0/). 